\title{
Modelando a Dispersão da Doença do Caranguejo Letárgico entre Estuários ${ }^{1}$
}

R.P. SANCHES', C.P. FERREIRA3, Departamento de Bioestatística IBB, UNESP

- Univ. Estadual Paulista, 18618-970 Botucatu, SP, Brasil.

\begin{abstract}
Resumo.Neste trabalho apresenta-se um modelo de mapas acoplados para o estudo da dispersão da Doença do Caranguejo Letárgico (DCL) entre dois estuários, em que mediu-se a influência da coleta de caranguejos e da existência de caranguejos resistentes sobre a dinâmica da doença. Observou-se que a coleta de caranguejos afeta tanto a intensidade quanto a periodicidade da doença e pode diminuir a probabilidade da doença se estabelecer. Porém, existe um limiar muito pequeno entre a não existência da doença no estuário onde há coleta e a extinção da população de caranguejos. Mostrou-se que a existência de caranguejos resistentes diminui a probabilidade de que a doença se estabeleça e que a migração do fungo pelo mar pode causar a sincronização das epidemias nos estuários.
\end{abstract}

Palavras-chave. Modelagem Matemática, Sincronização, Metapopulação.

\section{Introdução}

O caranguejo-uçá, Ucides cordatus, é encontrado desde Flórida (EUA) até Santa Catarina (Brasil). Constitui uma das principais fontes de renda das comunidades que vivem próximas aos manguezais, as quais dependem de sua comercialização como fonte de renda, utilizando-o também como alimento. Este crustáceo é um dos principais responsáveis pela decomposição da matéria orgânica nos estuários, e um biomonitor natural de áreas críticas, uma vez que demonstra sensibilidade a diversos poluentes [3].

Desde de 1997, observam-se eventos de mortandade do Ucides cordatus registrando-se uma diminuição de até $85 \%$ na taxa de captura do mesmo. Os caranguejos que residiam em regiões de mortandade, apresentavam sintomas semelhantes, a saber: letárgicos, sem controle das pernas e quelas, por isso deu-se a enfermidade, o nome de Doença do Caranguejo Letárgico (DCL). Vários experimentos foram realizados a fim de se identificar o que estava causando a doença nos caranguejos, mas somente em 2005 foi constatado que o agente causador da DCL é o fungo patogênico Exophiala cf psycrofila [2].

\footnotetext{
${ }^{1} \mathrm{MCT} / \mathrm{CNPq} 15 / 2007$

${ }^{2}$ rps@ibb.unesp.br; Programa de Mestrado em Biometria - bolsista CAPES.

3 pio@ibb.unesp.br, auxílio FAPESP 07/05265-1.
} 
Esta doença é especifica do Ucides cordatus, e é a primeira doença causada por fungos em crustáceos. Acredita-se que alguma espécie do fungo tenha se desenvolvido para uma feroz variedade, que parasita o caranguejo. $\mathrm{O}$ fungo causador da doença é dificilmente encontrado no período em que a doença não ocorre, e é provável que ele permaneça em caranguejos que não desenvolveram a doença (caranguejos resistentes), já que não são encontradas evidências do fungo no solo ou em plantas [1]. Aparentemente os caranguejos se infectam através do contado direto com o fungo. Caranguejos que desenvolvem a doença morrem em cerca de 9 à 35 dias após o contato com o patógeno. Como o sistema imunológico do caranguejo não possui memória imunológica, caranguejos resistentes voltam a ser suscetíveis. Nos eventos de mortandade só foram observados caranguejos adultos moribundos ou mortos, de ambos os sexos e em igual proporção. Como o fungo resiste a altas salinidades, uma das hipóteses é que a dispersão do mesmo ocorra pelo mar.

O objetivo desse trabalho é modelar a DCL, a fim de verificar o efeito causado pela migração de larvas de caranguejos e fungos entre estuários, a existência de caranguejos resistentes e a influência da coleta de caranguejos na dinâmica da doença. A apresentação está estruturada da seguinte forma: na seção 2 tem-se o modelo matemático da DCL, na seção 3 os resultados e discussão e por fim na seção 4 as conclusões.

\section{Modelo Matemático}

O modelo matemático apresentado a seguir é uma modificação do modelo proposto em [4], onde incluiu-se na dinâmica da doença, o compartimento de caranguejos jovens e a migração de caranguejos jovens (somente a larvas migram, $k_{1}<1$ ) e fungos, utilizando para isso a abordagem de mapas acoplados. Assim,

$$
\left\{\begin{array}{l}
\frac{d J_{i}}{d t}=\sum_{n} k_{1}\left(J_{n}-J_{i}\right)+\phi S_{i}-\left(\theta+\mu_{j}\right) J_{i} \\
\frac{d S_{i}}{d t}=\theta J_{i}-\beta \frac{F_{i} S_{i}}{K+S_{i}}-\left(\mu+\mu_{c_{i}}\right) S_{i}+\gamma_{i} I_{i}-c_{s} S_{i}^{2} \\
\frac{d I_{i}}{d t}=\beta \frac{F_{i} S_{i}}{K+S_{i}}-m I_{i} \\
\frac{d F_{i}}{d t}=\sum_{n} k_{2}\left(F_{n}-F_{i}\right)+\sigma \alpha I_{i}-\mu_{F} F_{i}
\end{array}\right.
$$

em que $m=\alpha+\gamma_{i}+\mu+\mu_{c_{i}}$, e $J_{i}, S_{i}, I_{i}, F_{i}$ denotam as populações de caranguejos jovens, caranguejos suscetíveis, caranguejos infectados e fungos respectivamente, sendo que os índices $i, n$ referem-se ao número do estuário (analisou-se a dinâmica da doença entre 2 estuários) e $i \neq n$.

A população de caranguejos jovens no estuário $i, J_{i}$, cresce (ou decresce) devido a migração de caranguejos entre os estuários a uma taxa $k_{1}$, cresce em razão da natalidade a uma taxa $\phi$, e decresce a uma taxa $\theta$ que corresponde aos caranguejos 
jovens que se tornam adultos e através da taxa de mortalidade $\mu_{j}$. A população de caranguejos suscetíveis no estuário $i, S_{i}$, cresce devido a passagem dos caranguejos jovens para a fase adulta e a existência de caranguejos resistentes, os quais entraram em contato com o fungo mas não desenvolveram a doença, representada por $\gamma_{i}$. Decresce a uma taxa de contato entre caranguejo e fungo dada por $\beta$, a qual depende da concentração de caranguejos no ambiente dada pelo parâmetro $K$ sendo, portanto, modelada pela equação de Michaelis-Menten. Diminui também a uma taxa de mortalidade $\mu+\mu_{c_{i}}$, onde a primeira representa a mortalidade natural e a segunda a coleta e, finalmente, pelo termo de competição $c_{s}$ entre caranguejos adultos. A população de caranguejos infectados no estuário $i, I_{i}$, cresce em função dos caranguejos que se tornam infectados e decresce pelas taxas de mortalidade devido à doença $\alpha$, caranguejos resistentes, caranguejos coletados e mortalidade natural. Finalmente, a população de fungos no estuário $i, F_{i}$, cresce (ou decresce) em função da migração de fungos a uma taxa $k_{2}$, cresce em razão da reprodução do fungo no organismo de caranguejos infectados a uma taxa $\sigma$ e decresce a uma taxa $\mu_{F}$ devido à mortalidade natural.

Na Tabela 1 tem-se a descrição de cada um dos parâmetros e o valor que os mesmos podem assumir. Supõe-se que os parâmetros $\gamma$ e $\mu_{c}$ podem assumir diferentes valores em cada um dos diferentes estuários e que os demais parâmetros são iguais nos dois estuários. Ao incluir o compartimento de caranguejos jovens (larvas mais juvenis), os quais não ficam doentes, assumiu-se que a taxa de encontro entre caranguejos suscetíveis e fungo é modelada pela equação de Michaelis-Menten, onde $K$ é o número de caranguejos por metro quadrado quando há $50 \%$ de chance de que ao entrar em contato com o fungo o caranguejo fique doente. Observou-se a necessidade de mudar a taxa de encontro entre fungo e caranguejo saudável (em [4] esta é modelada utilizando a lei de ação das massas) para que a periodicidade da doença observada nos estuários seja reproduzida pelo modelo. Considerou-se competição somente entre caranguejos adultos, visto que estes são territorialistas e que os mais jovens, em geral, ficam na região interna dos estuários (mais longe do mar) e não competem por fêmeas ou espaço. Em concordância com o modelo proposto em [4], o sistema (2.1) apresenta quatro cenários possíveis, equilíbrio trivial, equilíbrio livre da doença, equilíbrio endêmico, e ciclo limite que surge a partir da bifurcação de Hopf.

\subsection{Análise de estabilidade}

A determinação das soluções de equilíbrio e a análise de estabilidade destas, para o sistema acoplado, é difícil de ser obtida analiticamente (oito equações), de maneira que vamos supor que não há migração entre os estuários $\left(k_{1}=k_{2}=0\right)$ e tentaremos discutir os sistemas isolado.

Os valores de equilíbrio são obtidos igualando cada uma das derivadas do modelo (2.1) a zero. São eles:

a. Equilíbrio trivial denotado por $E_{0}=(0,0,0,0)$. Os autovalores obtidos a partir da construção do polinômio característico são dados por: $\lambda_{1}=-\mu_{F}$, $\lambda_{2}=-\left(\alpha+\mu+\mu_{c}+\gamma\right)$ e $\lambda_{3,4}=\frac{-\left(\mu+\theta+\mu_{j}+\mu_{c}\right) \pm \sqrt{x}}{2}$ em que, $x=\left(\mu_{j}+\mu_{c}+\theta+\right.$ 
Tabela 1: Parâmetros usados no modelo, descrição biológica e intervalo de valores possíveis.

\begin{tabular}{|c|c|c|}
\hline Taxas & Interpretação biológica (unidade) & Valores \\
\hline$\phi$ & natalidade dos caranguejos $\left(\right.$ dias $\left.^{-1}\right)$ & $0,25-0,34^{*}$ \\
\hline$k_{1}$ & migração do caranguejo jovem $\left(\operatorname{dias}^{-1}\right)$ & \\
\hline & $\left(\left(\mathrm{n}^{o}\right.\right.$ de caranguejos por $\left.\left.\mathrm{m}^{2}\right) \operatorname{dias}^{-1}\right)$ & - \\
\hline$\theta$ & transformação do caranguejo jovem & \\
\hline & em adulto $\left(\operatorname{dias}^{-1}\right)$ & $0,0011-0,0014^{*}$ \\
\hline$\mu_{j}$ & mortalidade do caranguejo jovem $\left(\right.$ dias $\left.^{-1}\right)$ & - \\
\hline$c_{s}$ & $\begin{array}{l}\text { competição entre caranguejos adultos } \\
\left(\left(\mathrm{n}^{o} \text { de caranguejos por } \mathrm{m}^{2}\right)^{-1} \text { dias }^{-1}\right)\end{array}$ & - \\
\hline$\mu_{c}$ & caranguejos coletados $\left(\operatorname{dias}^{-1}\right)$ & - \\
\hline$\mu$ & mortalidade do caranguejo adulto (dias $\left.{ }^{-1}\right)$ & $0,00028-0,0004^{*}$ \\
\hline$\beta$ & contato entre caranguejo e fungo (dias $\left.{ }^{-1}\right)$ & - \\
\hline$\alpha$ & mortalidade do caranguejo infectado $\left(\operatorname{dias}^{-1}\right)$ & $0,055-0,083^{*}$ \\
\hline$k_{2}$ & migração do fungo & \\
\hline & $\left(\left(\mathrm{n}^{o}\right.\right.$ de caranguejos por $\left.\mathrm{m}^{2}\right)$ dias $\left.^{-1}\right)$ & - \\
\hline$\gamma$ & caranguejos resistentes $\left(\operatorname{dias}^{-1}\right)$ & - \\
\hline$\sigma$ & $\begin{array}{l}\text { crescimento de fungos no interior de carangue- } \\
\text { jos infectados }\left(\operatorname{dias}^{-1}\right)\end{array}$ & - \\
\hline$\mu_{F}$ & mortalidade do fungo $\left(\operatorname{dias}^{-1}\right)$ & - \\
\hline
\end{tabular}

$\mu)^{2}-4\left(\left(\theta+\mu_{j}\right)\left(\mu+\mu_{c}\right)-\theta \sigma\right)$. Como $x>0, E_{0}$ é localmente assintoticamente estável se $\left(\theta+\mu_{j}\right)\left(\mu+\mu_{c}\right)<\theta \sigma$.

b. Equilíbrio livre da doença denotado por $E_{1}=(\bar{J}, \bar{S}, 0,0)$, sendo $\bar{J}=$ $\frac{\left(\mu+\mu_{c}+c_{s} \bar{S}\right) \bar{S}}{\theta}$ e $\bar{S}$ as soluções do polinômio $P(\bar{S})=\left(\theta+\mu_{j}\right) c_{s} \bar{S}^{2}-[\theta \phi-(\theta+$ $\left.\left.\mu_{j}\right)\left(\mu+\mu_{c}\right)\right] \bar{S}$. Observe que esta solução tem sentido biológico se $\bar{S}>0$, portanto $P(\bar{S})$ deve ter pelo menos uma solução positiva, o que permite definir $R_{c}=\frac{\theta \phi}{\left(\mu+\mu_{c}\right)\left(\theta+\mu_{j}\right)}$, então se $R_{c}>1, \bar{S}>0$.

A estabilidade local deste ponto de equilíbrio foi determinada utilizando o operador de próxima geração [9]. Neste caso, número de reprodutibilidade basal é definido como, $R_{0}=\rho\left(F V^{-1}\right)$ em que $\rho(A)$ é raio espectral da matriz A (seu autovalor dominante) e $F V^{-1}$ é o número esperado de novas infecções em um compartimento, produzidas pela chegada de um indivíduo infectado. A matriz $F$ corresponde aos termos responsáveis pelo aparecimento de novas infecções, e a matriz não singular $V$ corresponde aos termos de transição, assim

$$
F=\left[\begin{array}{cc}
0 & \frac{\beta \bar{S}}{K+S} \\
0 & 0
\end{array}\right], \quad V=\left[\begin{array}{cc}
\alpha+\gamma+\mu+\mu_{c} & 0 \\
-\sigma \alpha & \mu_{F}
\end{array}\right]
$$

Os autovalores da matriz da próxima geração $F V^{-1}$ são dados por $\lambda=0 \mathrm{e}$

$$
\lambda \equiv R_{0}=\frac{\alpha \sigma}{\mu_{F}}\left(\frac{\beta}{\left(\alpha+\gamma+\mu+\mu_{c}\right)} \frac{\bar{S}}{(K+\bar{S})}\right) .
$$


Portanto utilizando o Teorema 2 de [9], é possível afirmar que se $R_{0}<1$ este equilíbrio é localmente assintóticamente estável.

c. Equilíbrio endêmico denotado por $E_{3}=(\bar{J}, \bar{S}, \bar{I}, \bar{F})$, sendo, $\bar{S}=\frac{m \mu_{F} K}{\beta \alpha \sigma-m \mu_{F}}$, $\bar{J}=\frac{\phi \bar{S}}{\theta+\mu_{j}}, \bar{I}=\frac{\bar{S} \mu_{F}(K+\bar{S})\left[\left(\theta+\mu_{j}\right)\left(\mu+\mu_{c}+c_{s} \bar{S}\right)-\theta \phi\right]}{\left(\theta+\mu_{j}\right)\left(\gamma \mu_{F}(K+S)-\beta \alpha \sigma S\right)}$ e $\bar{F}=\frac{\alpha \sigma \bar{I}}{\mu_{F}}$. Este equilíbrio tem sentido biológico se $\bar{S}>0$ e $\bar{I}>0$. Para $\bar{S}>0$ deve-se ter que $\beta \alpha \sigma-m \mu_{F}>0$, então $R_{1}=\frac{\alpha \sigma}{\mu_{F}}\left(\frac{\beta}{\alpha+\mu+\mu_{c}+\gamma}\right)>1$. Para $\bar{I}>0$ são necessárias duas condições: $\left(\theta+\mu_{j}\right)\left(\mu+\mu_{c}+c_{s} \bar{S}\right)-\theta \phi>0$ que resulta em $R_{2}=\frac{\left(\theta+\mu_{j}\right)\left(\mu+\mu_{c}+c_{s} \bar{S}\right)}{\theta \phi}>1$ e $\gamma \mu_{F}(K+\bar{S})-\beta \alpha \sigma \bar{S}>0$ obtendo $R_{3}=\frac{\mu_{F}}{\alpha \sigma}\left(\frac{\gamma}{\beta} \frac{(K+\bar{S})}{S}\right)>1$. Neste caso, foi possível observar, através de simulações, que para uma pequena faixa de valores o equilíbrio endêmico é estável.

d. Ciclos limite, este cenário surge a partir da bifurcação de Hopf, e foi observado através de simulações numéricas.

\section{Resultados e Discussão}

Os resultados apresentados foram obtidos por meio de simulações utilizando o método Runge-Kutta de ordem 4 e linguagem de programação C. Em cada simulação, as condições iniciais foram fixadas em: 300 Jovens, 250 Suscetíveis, 0 Infectados, e 10 Fungos para o estuário 1 e 300 Jovens, 250 Suscetíveis, 0 Infectados, e 0 Fungos para o estuário 2. Os valores de alguns parâmetros foram mantidos constantes durante as simulações, são eles: $\beta=0,274 \operatorname{dias}^{-1}, \phi=0,34$ dias $^{-1}$, $\theta=0,0014 \operatorname{dias}^{-1}, \mu_{j}=0,015 \operatorname{dias}^{-1}, c_{s}=0,000001\left(\mathrm{n}^{o} \text { de indivíduos por } \mathrm{m}^{2}\right)^{-1}$ $\operatorname{dias}^{-1}, \mu=0,00028 \operatorname{dias}^{-1}, K=1 \mathrm{n}^{o}$ de caranguejos por $\mathrm{m}^{2}, \sigma=0.1265$ dias $^{-1}$, $\alpha=0,055 \operatorname{dias}^{-1}$ e $\mu_{F}=0,5 \operatorname{dias}^{-1}$.

\subsection{Migração de caranguejos jovens e fungos}

As larvas do Ucides cordatus, são depositadas no mar pelas fêmeas do caranguejo e seu desenvolvimento compreende de 5 à 6 estágios larvais da fase zoea, em um período de de 3 à 5 semanas [8, 5]. A migração do caranguejo jovem foi incorporada ao modelo, acontecendo durante os primeiros 30 dias de cada ano, em ambos sentidos, e é proporcional a quantidade de caranguejos jovens, sendo a constante de proporcionalidade dada por $k_{1}=0,01 \mathrm{n}^{o}$ de indivíduos por $\mathrm{m}^{2} \operatorname{dias}^{-1}$. Considerase que o compartimento dos caranguejos jovens engloba as larvas do caranguejo e os caranguejos juvenis, os quais ainda não participam do processo de reprodução (acasalamento). Em particular, somente as larvas migram de um estuário a outro.

Ao se cultivar o fungo em diferentes níveis de salinidade, constatou-se que o mesmo sobrevive, mesmo a uma alta taxa de salinidade, por um extenso período de tempo, reforçando a idéia de que a dispersão do fungo ocorra pelo mar [7]. A migração do fungo foi incorporada ao modelo, acontecendo duas vezes por mês, em ambos sentidos, a fim de imitar a maneira com a qual a doença vem se dispersando, 
sentido norte-sul e sul-norte [1]. Para isto foi suposto que o fungo migra junto com as marés altas, que acontecem quinzenalmente nas luas nova e cheia.

Se não há migração do fungo tem-se que a doença ocorre em apenas um dos estuários (estuário 1 - condição inicial dada). Após a inclusão da migração de fungos, os dois estuários apresentam a doença, como pode ser observado na Figura 1, onde tem-se, respectivamente, nas linhas contínua e tracejada as populações de suscetíveis e infectados do estuário 1 , e em $(\circ)$ e $(\bullet)$ populações de suscetíveis e infectados do estuário 2. A migração de fungos é proporcional a quantidade de fungos nos dois estuários, sendo a constante de proporcionalidade dada por $k_{2}=0,05 \mathrm{n}^{o}$ de indivíduos por $\mathrm{m}^{2} \operatorname{dias}^{-1}$.

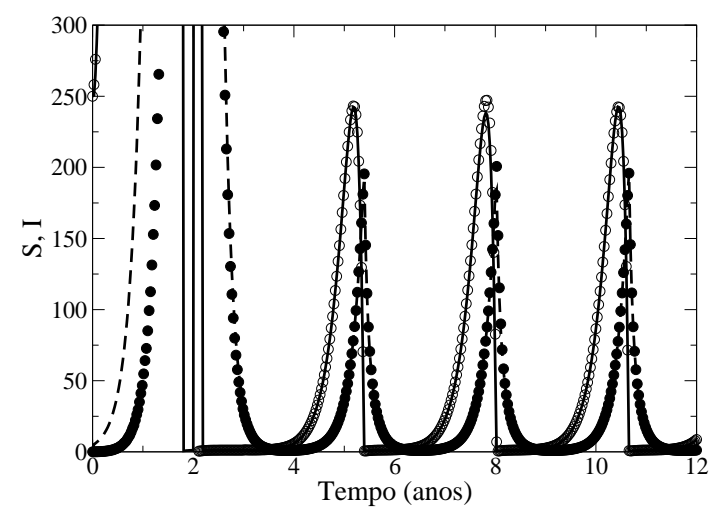

Figura 1: Dinâmica da doença nos dois estuários. Nas linhas contínua e tracejada as populações de suscetíveis e infectados do estuário 1 , os símbolos (०) e (•) correspondem, respectivamente, as populações de suscetíveis e infectados do estuário 2.

Devido a migração de fungos, o aparecimento dos picos epidêmicos nos estuários é sincronizado, isto é, após um período de transiente as populações dos estuários oscilam com o mesmo padrão temporal.

\subsection{Caranguejos coletados}

As populações que vivem próximas aos mangues geralmente têm como fonte de renda a exploração dos manguezais onde uma das principais atividades é a coleta de caranguejos. A coleta pode ser feita em um determinado período do ano ou no ano inteiro [6]. Foram abordadas, via simulação, essas duas estratégias de coleta de caranguejos, em um primeiro momento coletou-se a mesma quantidade de caranguejos nos dois estuários, e no segundo momento coletou-se caranguejos em apenas um dos estuários. Para a coleta periódica, a retirada de caranguejos foi feita durante 9 meses de cada ano, iniciando a coleta no segundo mês do ano (após o término da migração das larvas), para isto foi suposto que neste caso, estes "caranguejeros"respeitam o período de defeso, época do ano em que é proibida a caça do caranguejo devido ao seu período de reprodução, esse período não tem uma duração específica e muda conforme a região. 
Na Figura 2, foi calculada a razão entre as áreas da curva de infectados versus tempo e suscetíveis versus tempo em função de $\mu_{c}$ quando a coleta periódica é feita nos dois estuários. Observa-se que, para $\mu_{c}<0,02$ tem-se equilíbrio endêmico e a medida que $\mu_{c}$ aumenta, a razão entre a curva de infectados versus tempo e suscetível versus tempo diminui, para $0,02 \leq \mu_{c}<0,047$ tem-se o equilíbrio livre da doença. Finalmente, para $\mu_{c} \geq 0,047$ a população de caranguejos se extingue nos 2 estuários. O formato irregular da curva deve-se a coleta periódica de caranguejos.

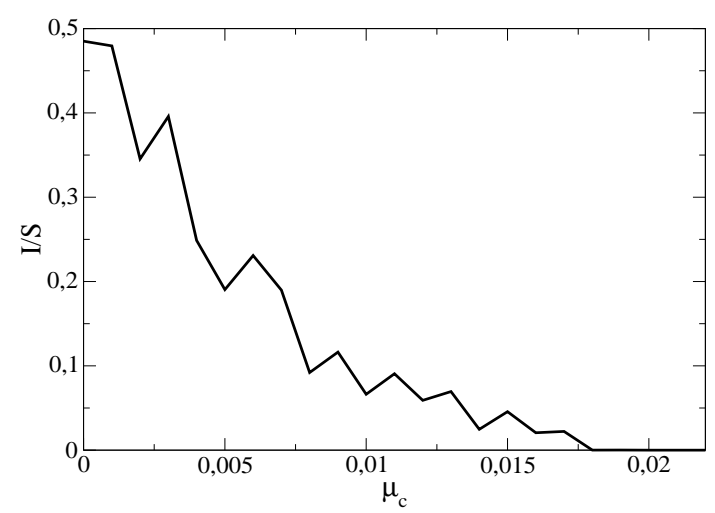

Figura 2: Razão entre as áreas de infectados e suscetíveis versus taxa de coleta dos caranguejos, $\mu_{c}$.

Na Figura 3 tem-se a dinâmica da doença quando a coleta periódica é feita em apenas um estuário (estuário 2), tem-se nas linhas contínua e tracejada os estuários 1 e 2 respectivamente. O estuário 2 apresenta uma quantidade menor de caranguejos infectados quando comparado ao estuário 1 devido a coleta, porém o estuário 1 também apresenta uma redução em sua população de caranguejos infectados em razão da redução de sua população de fungos como resultado da migração entre estuários. É possível verificar este fato comparando as razões entre infectados e suscetíveis, quando não havia coleta de caranguejos, a razão de infectados em relação aos suscetíveis (a razão das áreas da curva de infectado versus tempo e suscetível versus tempo) era de 0,49, e após a coleta passou a ser de 0,25. Neste caso os equilíbrios livre da doença e trivial não são obtidos mesmo para altos valores de $\mu_{c_{2}}$.

Na Tabela 3.2., tem-se as médias, desvios padrões e os coeficientes de variação em relação aos picos de infecção medidos para $t \in[30,50]$ anos (após o transiente e durante um intervalo de tempo arbitrário). O número médio de infectados do estuário 1 é aproximadamente 9 vezes maior que o estuário 2 (a razão das áreas da curva de infectado versus tempo e suscetível versus tempo para o estuário 1 e 2 são respectivamente, 0,25 e 0,09), o coeficiente de variação do estuário 1 é menor do que $1 \%$, e o coeficiente de variação do estuário 2 é de 4,59\%. Ao se analisar o gráfico da evolução temporal da população de infectados no tempo, percebeu-se que no caso do estuário 2 tem-se picos anuais de diferentes intensidades, porém ao 


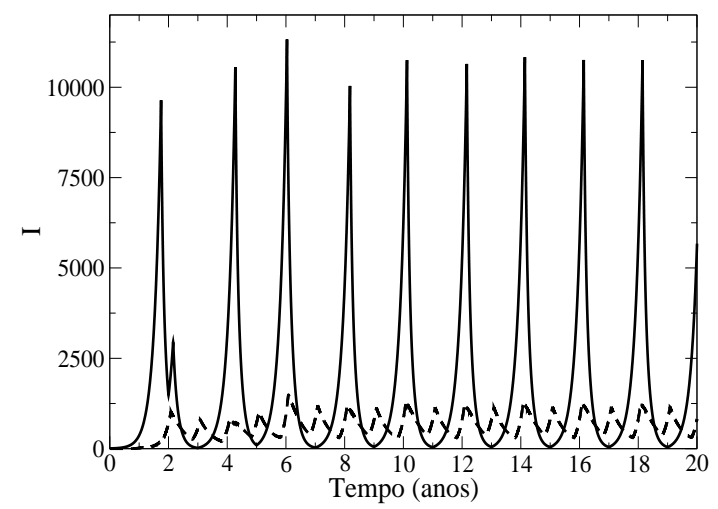

Figura 3: Evolução temporal da população de caranguejos infectados para coleta periódica com $\mu_{c_{1}}=0$ dias $^{-1}$ no estuário 1 (linha contínua) e $\mu_{c_{2}}=0,02$ dias $^{-1}$ no estuário 2 (linha tracejada).

verificar os valores assumidos por cada pico notou-se que eles se repetem a cada 6 anos, o que explica o coeficiente de variação maior.

Tabela 2: Picos de infecção em relação a Figura 3.

\begin{tabular}{cccccc}
\hline & & $\mathrm{n}^{o}$ de picos & média & desvio padrão & coeficiente de variação(\%) \\
\hline$\mu_{c_{1}}$ & 0 & 10 & 10752,58 & 25,04 & 0,23 \\
$\mu_{c_{2}}$ & 0,02 & 20 & 1212,46 & 55,62 & 4,59 \\
\hline
\end{tabular}

Na Tabela 3, tem-se as médias, desvios padrões e os coeficientes de variação em relação as distâncias entre os picos de infecção medidos para $t \in[30,50]$. Pode-se notar que a coleta periódica afeta a periodicidade da doença, pois para o conjunto de parâmetros fixados, sem a coleta periódica a distância média entre os picos era de aproximadamente 2,7 anos, e com a coleta periódica ela passou a ser de 2 anos para o estuário 1 e de 1 ano para o estuário 2, e o coeficiente de variação está abaixo de $1 \%$ nos dois estuários indicando um baixo desvio padrão.

Tabela 3: Distâncias entre os picos em relação a Figura 3.

\begin{tabular}{ccccc}
\hline & & média & desvio padrão & coeficiente de variação(\%) \\
\hline$\mu_{c_{1}}$ & 0 & 2,000 & 0,007 & 0,333 \\
$\mu_{c_{2}}$ & 0,02 & 1,000 & 0,003 & 0,334 \\
\hline
\end{tabular}

Na Figura 4, foi calculada a razão entre as áreas da curva de infectados versus tempo e suscetíveis versus tempo em função de $\mu_{c}$ quando a coleta contínua é feita nos dois estuários. A medida que $\mu_{c}$ aumenta, a razão entre a curva de infectados 
versus tempo e suscetível versus tempo diminui, para $0,015 \leq \mu_{c}<0,031$ tem-se o equilíbrio livre da doença. Finalmente, para $\mu_{c} \geq 0,031$ a população de caranguejos se extingue nos 2 estuários.

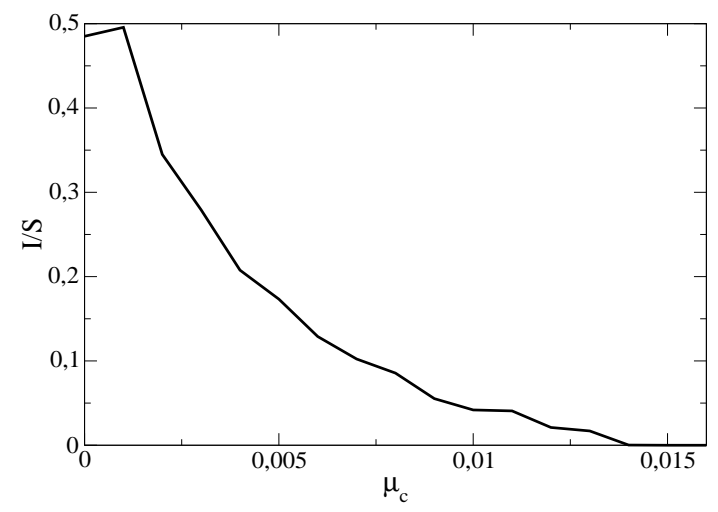

Figura 4: Razão entre as áreas de infectados e suscetíveis versus taxa de coleta de caranguejos, $\mu_{c}$.

Na Figura 5 tem-se a dinâmica da doença quando a coleta contínua é feita em apenas um estuário (estuário 2), tem-se nas linhas contínua e tracejada os estuários 1 e 2 respectivamente. Como no caso da coleta periódica a população de caranguejos infectados do estuário 2 é menor do que a população de caranguejos infectados do estuário 1, e existe uma diminuição na população de infectados do estuário 1, quando comparado à situação em que não haviam caranguejos coletados em nenhum dos estuários, devido ao mesmo motivo do caso da coleta periódica. Comparando as razões entre suscetíveis e infectados, tem-se que quando não havia coleta de caranguejos a razão de infectados, em relação aos suscetíveis era de 0,49, e após a coleta passou a ser de 0,24 . Mesmo para altos valores de $\mu_{c_{2}}$ tem-se o equilíbrio endêmico.

Na Tabela 4, tem-se as médias, desvios padrões e os coeficientes de variação em relação aos picos de infecção medidos para $t \in[30,50]$. Neste caso, o número médio de infectados do estuário 1 é aproximadamente 14 vezes maior que o número médio de infectados do estuário 2, e o coeficiente de ambos estuários é menor do que $1 \%$ indicando um baixo desvio padrão.

Tabela 4: Picos de infecção em relação a Figura 5

\begin{tabular}{lccccc}
\hline & & $\mathrm{n}^{\circ}$ de picos & média & desvio padrão & coeficiente de variação(\%) \\
\hline$\mu_{c_{1}}$ & 0 & 10 & 10408,5 & 71,8 & 0,7 \\
$\mu_{c_{2}}$ & 0,015 & 10 & 747,1 & 5,4 & 0,7 \\
\hline
\end{tabular}

Na Tabela 5, tem-se as médias, desvios padrões e os coeficientes de variação em relação as distâncias entre os picos de infecção, medidos para $t \in[30,50]$. Neste caso, a média entre as distâncias temporais não apresentam diferença entre si, e seus 


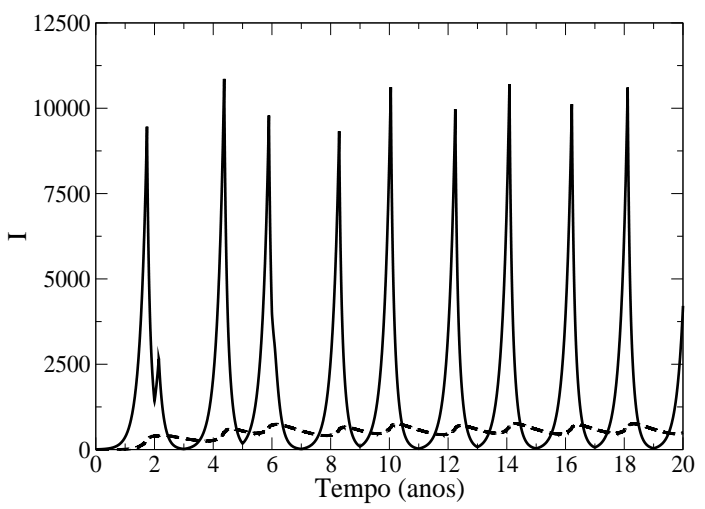

Figura 5: Evolução temporal da população de caranguejos infectados para coleta contínua com $\mu_{c_{1}}=0$ dias $^{-1}$ no estuário 1 (linha contínua) e $\mu_{c_{2}}=0,02 \operatorname{dias}^{-1}$ no estuário 2 (linha tracejada).

coeficientes de variação são menores do que $2 \%$ indicando um baixo desvio padrão.

Tabela 5: Distâncias entre os picos em relação a Figura 5

\begin{tabular}{lcccc}
\hline & & média & desvio padrão & coeficiente de variação(\%) \\
\hline$\mu_{c_{1}}$ & 0 & 2,00 & 0,03 & 1,61 \\
$\mu_{c_{2}}$ & 0,015 & 2,00 & 0,03 & 1,70 \\
\hline
\end{tabular}

No modelo proposto em [4] para $\mu_{c} \geq 0,4$ obtem-se o equilíbrio trivial e para $\mu_{c}<0,4$ a maioria das soluções são periódicas. No presente estudo, para a coleta periódica, foi obtido que se $\mu_{c}<0,02$ tem-se soluções periódicas, para $0,02 \leq \mu_{c}<$ 0,047 é obtido o equilíbrio livre da doença e se $\mu_{c} \geq 0,047$ é obtido o equilíbrio trivial. No caso da coleta contínua, observa-se que para $\mu_{c}<0,015$ tem-se soluções periódicas, para $0,015 \leq \mu_{c}<0,031$ tem-se o equilíbrio livre da doença, e se $\mu_{c} \geq 0,031$ tem-se o equilíbrio trivial.

\subsection{Caranguejos resistentes}

Existem caranguejos que entram em contato com o fungo, porém não desenvolvem a doença e são chamados de caranguejos resistentes. Como os artrópodes, em geral, não possuem memória imunológica, eles voltam a ser suscetíveis. O motivo pelo qual alguns caranguejos conseguem neutralizar a ação do fungo em seu organismo e outros não, é mais um dos aspectos desconhecidos dessa doença [5]. Esta abordagem tem por finalidade estudar o efeito causado pela existência de caranguejos resistentes nos estuários. Fez-se duas abordagens: a existência de caranguejos resistentes nos dois estuários, e a existência de caranguejos resistentes em apenas um estuário.

Na Figura 6, foi calculada a razão entre as áreas da curva de infectados versus 
tempo e suscetíveis versus tempo em função de $\gamma$. Para $\gamma \geq 0,015$ tem-se o equilíbrio livre da doença.

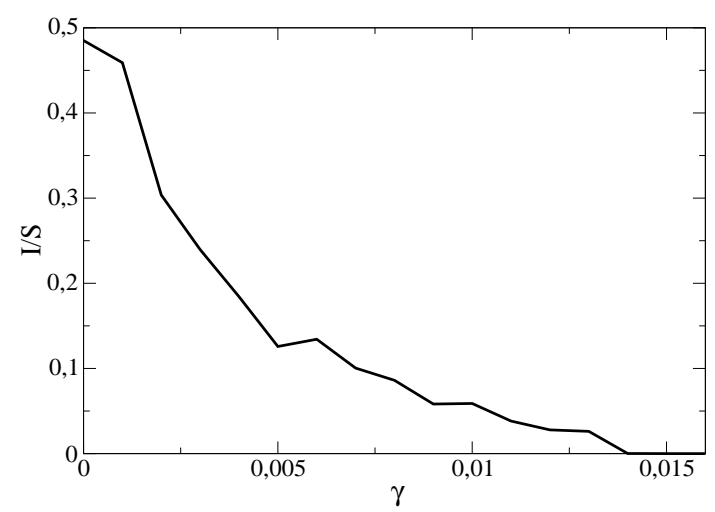

Figura 6: Razão entre as áreas de infectados e suscetíveis versus $\gamma$.

A Figura 7 mostra a dinâmica da doença em que existem caranguejos resistentes em apenas um dos estuários (estuário 2), onde as linhas contínua e tracejada representam os estuários 1 e 2 respectivamente. Como no caso dos caranguejos coletados, o estuário 2 possui um número menor de infectados quando comparado ao estuário 1, e, por sua vez, o estuário 1 possui uma proporção menor de infectados, quando comparado ao caso em que não haviam caranguejos resistentes em nenhum dos estuários. Neste caso a razão de infectados era de 0,49 e após a inclusão de caranguejos resitentes no estuário 2 essa razão passou a ser de 0.28 .

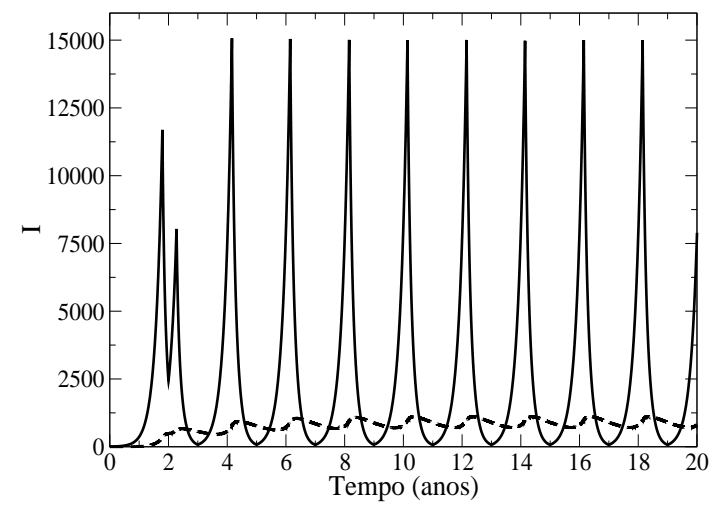

Figura 7: Caranguejos resistentes com $\gamma_{1}=0 \operatorname{dias}^{-1}$ no estuário 1 (linha contínua) e $\gamma_{2}=0,015 \operatorname{dias}^{-1}$ no estuário 2 (linha tracejada).

A Tabela 6 mostra os valores das médias, desvios padrões e coeficientes de variação dos estuários 1 e 2 da Figura 7 medidos para $t \in[30,50]$ em relação aos picos 
de infecção. A média de caranguejos infectados do estuário 1 é, aproximadamente, 13,5 vezes maior do que do estuário 2, e o coeficiente de variação dos dois estuários é menor do que $1 \%$ indicando um baixo desvio padrão.

Tabela 6: Picos de infecção da Figura 7

\begin{tabular}{cccccc}
\hline & & $\mathrm{n}^{o}$ de picos & média & desvio padrão & coeficiente de variação(\%) \\
\hline$\gamma_{1}$ & 0 & 10 & 14996,50 & 9,24 & 0,06 \\
$\gamma_{2}$ & 0,015 & 10 & 1118,83 & 1,68 & 0,15 \\
\hline
\end{tabular}

A Tabela 7 mostra os valores das médias, desvios padrões e coeficientes de variação dos estuários 1 e 2 da Figura 7 medidos para $t \in[30,50]$ em relação às distâncias entre os picos, onde pode ser visto que suas médias são iguais e seus coeficientes de variação estão abaixo de $1 \%$ indicando um baixo desvio padrão.

Tabela 7: Distâncias entre os picos da Figura 7

\begin{tabular}{|c|c|c|c|c|}
\hline & & média & desvio padrão & coeficiente de variação(\%) \\
\hline$\gamma_{1}$ & 0 & 2,00 & 0,01 & 0,28 \\
\hline$\gamma_{2}$ & 0,015 & 2,00 & 0,02 & 0,97 \\
\hline
\end{tabular}

No modelo proposto em [4], para $\gamma>0,03$ não são possíveis soluções periódicas e o sistema pode tanto alcançar o equilíbrio endêmico, quanto o livre da doença. No entanto a probabilidade de se ter o equilíbrio livre da doença é muito baixa. No modelo apresentado neste estudo, tem-se que, para $\gamma<0,015$ são obtidas soluções periódicas e para $\gamma \geq 0,015$ tem-se o equilíbrio livre da doença. A diferença obtida entre os dois modelos se deve a suposição feita com relação à probabilidade de contato entre caranguejos e fungos, a qual foi modela em [4] como proporcional ao tamanho das duas população (lei de ação das massas) e neste trabalho pela equação de Michaelis-Menten. Uma pergunta fica em aberto: qual das duas suposições é a mais correta? Porém para respondê-la são necessárias evidências experimentais.

\section{Conclusão}

O modelo proposto reproduz os padrões da DCL e possibilita o estudo da migração dos caranguejos jovens e fungos, o efeito da retirada de caranguejos dos estuários e a existência de caranguejos resistentes na dinâmica da transmissão da doença.

A coleta de caranguejos diminui a probabilidade da doença se estabelecer no estuário. Para o conjunto de parâmetros utilizado, se a coleta é feita periodicamente nos dois estuários, para $0,02 \leq \mu_{c}<0,047$ tem-se o equilíbrio livre da doença e se $\mu_{c} \geq 0,047$ é obtido o equilíbrio trivial, entretanto, se a retirada é feita em apenas um estuário obtém-se o equilíbrio endêmico. Se a coleta é contínua, para $0,015 \leq \mu_{c}<0,031$ tem-se o equilíbrio livre da doença e se $\mu_{c} \geq 0,031$ obtem-se o equilíbrio trival, da mesma forma anterior, se a coleta é feita em apenas um dos estuários mantêm-se o equilíbrio endêmico. Observa-se que nos dois casos a partir de um certo valor de $\mu_{c}$ tem-se o equilíbrio trivial indicando que a ação da coleta 
combinada com a doença pode levar a extinção dos caranguejos. A análise dos desvios padrões e coeficientes de variação permitiram concluir que, quando a coleta é feita em apenas um dos estuários, ela afeta tanto a intensidade da doença, quanto sua periodicidade.

A existência de caranguejos resistentes também diminui a probabilidade de que a doença se estabeleça no estuário. Para o conjunto de parâmetros utilizado, se existem caranguejos resistentes nos dois estuários, tem-se o equilíbrio livre da doença para $\gamma \geq 0,015$. Porém, se existem caranguejos resistentes em apenas um estuário obtém-se o equilíbrio endêmico. A análise dos desvios padrões e coeficientes de variação permitiram concluir que, quando existem caranguejos resistentes em apenas um dos estuários, o número de caranguejos infectados decresce, e os picos de infecção acontecem com maior frequência.

No modelo proposto por [4], estudou-se os padrões temporais apresentados pela doença e a influência causada pela variação de alguns parâmetros, como taxa de coleta, taxa de contato e outros, na dinâmica da mesma em um único estuário. $\mathrm{O}$ modelo de três equações diferencias ordinárias descreve a variação das populações de caranguejos saudáveis, caranguejos infectados e fungo. O encontro entre as populações de caranguejos saudáveis e fungo foi modelado através da lei de ação das massas e observou-se quatro situações distintas para a dinâmica da doença: o equilíbrio trivial, o equilíbrio livre da doença, o equilíbrio endêmico e o ciclo limite o qual surge de uma bifurcação de Hopf. No presente estudo foram incluídos ao modelo o compartimento de caranguejos jovens, e migração de caranguejos jovens e fungos. Além disso, utilizando a abordagem de maplas acoplados, o comportamento da doença em estuários distintos e acoplados foi estudada. Mostrou-se que intervenções em um estuário afetam a dinâmica deste e do estuário vizinho devido ao acoplamento entre eles. Por exemplo, o modelo prevê a sincronização da doença nos estuários devido a migração do fungo. Na prática, existem vários estuários acoplados, com características próprias, de maneira que o padrão espaço-temporal da doença é diferente nos vários estuários e fenômenos de sincronização ainda não foram observados. Esta heterogeneidade espacial é facilmente reproduzida pelo modelo de mapas acoplados (aumentando o número de estuários e diferenciando-os com relação aos parâmetros do modelo), de maneira que dados experimentais, por exemplo, indicência da doença em cada um dos estuários, podem ser utilizados para tornar o modelo mais realista.

Comparando os resultados obtidos neste estudo com os resultados obtidos em [4], observa-se que em relação a coleta de caranguejos, os dois modelos apresentam um valor limiar de $\mu_{c}$ a partir do qual obtem-se o equilíbrio trivial, e para valores abaixo desse valor a maioria das soluções são periódicas. No caso da existência de caranguejos resistentes, no modelo proposto por [4], tem-se que a probabilidade de obter-se o equilíbrio livre da doença é muito baixa, enquanto neste estudo, a partir de um valor limiar de $\gamma$, este equilíbrio é obtido. Esta diferença se deve a suposição feita em relação ao contato entre caranguejos e fungos, o qual foi modelado em [4] proporcionalmente ao tamanho das populações de caranguejos e fungos (lei de ação das massas), e neste trabalho foi modelado pela equação de Michaelis-Menten. 


\title{
Agradecimentos
}

Aos revisores da revista TEMA cujos comentários contribuiram para a qualidade da escrita, discussão e apresentação deste trabalho.

\begin{abstract}
In this paper a coupled map model to study the dispersion of DCL of two estuaries was developed. Using this model, the importance of crabs collection and also the presence of resistant crabs in estuaries on disease dynamics was assessed. The results shown that crabs collection affects both intensity and disease frequency and may decrease the disease probability of establishment in an estuary. However, the threshold between disease free-equilibrium and crabs extinction is small. Finally, it was shown that the existence of resistant crabs can also decreases disease probability of establishment, and fungus migration can cause disease epidemics to become synchronized in estuaries.
\end{abstract}

\section{Referências}

[1] W.A. Boeger, M.R. Pie, Montando o quebra cabeça, Revista do GIA, 1, No. 2 (2006), 34-36.

[2] W.A. Boeger, M.R. Pie, A. Ostrensky, L. Patella, Lethargic crab disease:multidiciplinary evidence supports a mycotic etiology, Mem. Inst. Oswaldo cruz, 100, No.2 (2005), 161-167.

[3] G. Castilho, Ele vive na lama, mas tem sangue azul, Revista do GIA, 1, No. 2 (2006), 18-19.

[4] C.P. Ferreira, M.R. Pie, L. Esteva, P.F. Mancera, W.A. Boeger, A. Ostrensky, Modelling the lethargic crab disease, Journal of Biological Dynamics, 3, No. 2 (2009), 620-634.

[5] Grupo Integrado de Aquicultura (GIA), Desvendando uma tragédia nos manguezais brasileiros, Revista do GIA, 1, No. 2 (2006).

[6] M. Jankowisky, J.S.R. Pires, N. Nordi, Contribuição ao manejo participativo do caranguejo-uçá, Ucides Cordatus (L.1763) em Cananéia-SP, Boletim do Instituto de Pesca, 32, No. 2 (2006), 221-228.

[7] R.O. Ribeiro, "Doença do Caranguejo Letárgico: Desvendando questões etiológicas, epidemiológicas e de Saúde Pública", Dissertação de Mestrado, Universidade Federal do Paraná, Curitiba, PR, 2008.

[8] D.J.B. Simith, K. Diele, Metamorphosis of mangrove crab megalopae, Ucides cordatus (Ocypodidae): Effects of interspecific versus intraspecific settlement cues, Journal of Experimental Marine Biology and Ecology, 362, No. 2 (2008), 101-107.

[9] P. Van Den Driessche, J. Watmough, Reproduction numbers and sub-threshold endemic equilibria for compartimental models of disease transmission, Mathematical Biosciences,180, (2002), 29-48. 